\title{
Modal representation of spatial coherence in dissipative and resonant photonic systems
}

\author{
C. Sauvan,,${ }^{1, *}$ J. P. Hugonin, ${ }^{1}$ R. Carminati, ${ }^{2}$ and P. Lalanne ${ }^{3}$ \\ ${ }^{1}$ Laboratoire Charles Fabry, Institut d'Optique, CNRS, Université Paris Sud, 2 avenue Augustin Fresnel, 91127 Palaiseau, France \\ ${ }^{2}$ Institut Langevin, ESPCI ParisTech, CNRS, 1 rue Jussieu, 75238 Paris Cedex 05, France \\ ${ }^{3}$ Laboratoire Photonique Numérique et Nanosciences, Institut d'Optique, Université Bordeaux, CNRS, 33405 Talence, France
}

(Received 18 January 2014; published 16 April 2014)

\begin{abstract}
We provide a self-consistent electromagnetic theory of the link between spatial coherence and optical resonances in three-dimensional open and dissipative photonic systems. The theory that relies on the concept of quasinormal modes with complex frequencies provides an accurate modal expansion of the imaginary part of the Green tensor that correctly treats the effects of radiative leakage, absorption, and dispersion. It represents a powerful tool for calculating and understanding the degree of spatial coherence in complex photonic or plasmonic systems that are governed by a small number of resonances. Comparisons with fully vectorial calculations evidence the high accuracy of the predictions achieved by our semianalytical treatment in the case of coupled photonic-crystal microcavities and plasmonic nanoantennas made of metallic nanorods.
\end{abstract}

DOI: 10.1103/PhysRevA.89.043825

PACS number(s): 42.25.Dd, 42.50.Pq, 42.70.Qs, 78.67.-n

\section{INTRODUCTION}

The development of micro- and nanotechnologies has recently opened a wide range of possibilities for controlling light at the wavelength scale or below. Fine control of the light - emission, transport, and detection —in small volumes is at the heart of various applications, such as high-performance sensors [1,2], light focusing below the diffraction limit $[3,4]$, nanolasers [5,6], solid-state single-photon sources [7,8], or photovoltaic devices [9]. Most of these applications rely on the use of optical resonances, which can be either localized in small volumes or delocalized over the system. In addition to the resonant character of the electromagnetic field, a broad range of processes also takes advantage of spatial coherence, including coherent control at the nanoscale [10], superradiance [11-13], and spatial focusing by time reversal or phase conjugation [14]. Clearly, precise knowledge of how the spatial extent of the resonances drives the coherence properties of the system is of central importance for further developments.

In a number of situations where spatial coherence plays an important role, the imaginary part of the Green tensor $\mathbf{G}$ at two points invariably appears in the equations [15-18]. Consider, for instance, the emission of two coherent point dipoles $\mathbf{p}_{1}$ and $\mathbf{p}_{2}$ located at $\mathbf{r}_{1}$ and $\mathbf{r}_{2}$. The total emitted power is given by $\Gamma_{1}+\Gamma_{2}+\mu_{0} \omega^{3} \operatorname{Re}\left[\mathbf{p}_{1}^{*} \cdot \operatorname{Im} \mathbf{G}\left(\mathbf{r}_{1}, \mathbf{r}_{2}, \omega\right) \mathbf{p}_{2}\right]$, where $\omega$ is the frequency and $\Gamma_{i}=0.5 \mu_{0} \omega^{3} \operatorname{Im}\left[\mathbf{p}_{i}^{*} \cdot \mathbf{G}\left(\mathbf{r}_{i}, \mathbf{r}_{i}, \omega\right) \mathbf{p}_{i}\right]$ is the power emitted by $\mathbf{p}_{i}$ alone. This simple expression highlights the key role played by the imaginary part of the Green tensor in the phenomenon of superradiance $[12,13]$. Other coherent processes are also driven by $\operatorname{Im}(\mathbf{G})$, such as the process of focusing by time reversal in a closed cavity [19].

From this observation, Cazé et al. have recently proposed the concept of cross density of states (CDOS) to characterize the spatial coherence of a complex photonic or plasmonic system [20]. The CDOS is the two-point quantity $\rho\left(\mathbf{r}, \mathbf{r}^{\prime}, \omega\right)$ defined as

$$
\rho\left(\mathbf{r}, \mathbf{r}^{\prime}, \omega\right)=\frac{2 \omega}{\pi c^{2}} \operatorname{Im}\left[\operatorname{Tr} \mathbf{G}\left(\mathbf{r}, \mathbf{r}^{\prime}, \omega\right)\right]
$$

\footnotetext{
*christophe.sauvan@institutoptique.fr
}

In this expression, $c$ is the speed of light in vacuum, $\mathbf{G}\left(\mathbf{r}, \mathbf{r}^{\prime}, \omega\right)$ is the electric dyadic Green function, and $\operatorname{Tr}$ denotes the trace of a tensor. The Green function is defined from the electric field created at point $\mathbf{r}$ by an electric-dipole source $\mathbf{p}$ at point $\mathbf{r}^{\prime}$ through the relation $\mathbf{E}(\mathbf{r})=\mu_{0} \omega^{2} \mathbf{G}\left(\mathbf{r}, \mathbf{r}^{\prime}, \omega\right) \mathbf{p}$. This definition of the CDOS has been chosen so that it reduces to the electromagnetic local density of states (LDOS) when $\mathbf{r}=\mathbf{r}^{\prime}$. Note also that reciprocity imposes $\rho\left(\mathbf{r}, \mathbf{r}^{\prime}, \omega\right)=\rho\left(\mathbf{r}^{\prime}, \mathbf{r}, \omega\right)$.

In the case of a closed and nonabsorbing system, the relation between the CDOS and the resonances can be easily derived. In this ideal conservative case (Hermitian system), a discrete orthonormal basis of eigenmodes $\mathbf{E}_{m}(\mathbf{r})$ with real eigenfrequencies $\omega_{m}$ can be introduced (the so-called normal modes [21]) and the CDOS defined by Eq. (1) can be rewritten as [20]

$$
\rho\left(\mathbf{r}, \mathbf{r}^{\prime}, \omega\right)=\sum_{m} \operatorname{Tr}\left[\mathbf{E}_{m}(\mathbf{r}) \otimes \mathbf{E}_{m}^{*}\left(\mathbf{r}^{\prime}\right)\right] \delta\left(\omega-\omega_{m}\right),
$$

where $\delta$ is the Dirac distribution and $\otimes$ denotes the tensor (or dyadic) product between two vectors. Such an expansion onto the system resonances is extremely useful both from a physical and from a practical point of view. First, a modal expansion allows for deep physical insight into the problem since it explicitly shows that the CDOS gives a measure of the number of channels (modes) connecting two points in the system, weighted by their strength at both point $\mathbf{r}$ and point $\mathbf{r}^{\prime}$. In addition, since it can be handled analytically once the modes have been calculated, a modal expansion also enables very efficient calculations specifically tailored for design purposes or fast optimizations.

Unfortunately, in most cases of interest for photonics, we are concerned with open systems that lose energy by radiation and that are composed of absorbing and dispersive materials such as metals or semiconductors. In the case of such a dissipative (non-Hermitian) system, the eigenmodes are no longer normal modes and the modal expansion given by Eq. (2) is not valid. One usual resolution to bypass this issue is to use a phenomenological approach in which energy dissipation is introduced by broadening the eigenmodes with a quality factor $Q_{m}$ and replacing the Dirac $\delta$ function in Eq. (2) with a Lorentzian line shape. However, if this trick works for 
resonators with a small leakage (i.e., a high quality factor), it becomes largely unsubstantiated for less confined resonances characterized by low quality factors [22-24].

Actually, in the case of an open, absorbing, and dispersive system, the rigorous introduction of a set of eigenmodes is more involved than a simple extension of the lossless case. Perhaps one of the greatest difficulty arises from the absence of closed boundaries. This is a well-known issue that has been documented in various area of wave physics, from the scattering by a potential in quantum mechanics [25-28] to optical microcavities [29-35], plasmonics [24,36], and acoustics [37].

An adequate modal representation of a dissipative resonant system should rely on the natural eigenmodes of the structure. The latter are no longer normal modes with real eigenfrequencies but, rather, poles of the scattering matrix associated with complex eigenfrequencies. Consistent with the literature on open optical cavities [29,30], we refer to these modes as quasinormal modes (QNMs). The eigenmodes of open resonant systems are also known in the literature as decaying states [26], resonant states [27,34], and leaky modes [38]. It is worth mentioning that, for some particular or limiting cases, different modal representations that do not involve QNMs have been developed; see, for instance, in the quasistatic limit, the use of the eigenmodes of a Poisson equation for the electrostatic potential $[39,40]$ or the introduction of "constant-flux states" for two-dimensional (2D) media in transverse electric polarization [41].

The question of the spectral representation of waves as a superposition of QNMs has a venerable history [25-35,42-46]. However, although light interaction with resonant modes is a cornerstone of optics [47], QNM concepts are not widely used for analyzing photonic structures. Perhaps this is due to the fact that, in contrast to normal modes, QNMs are no longer of finite energy since they grow exponentially in space at infinity. Important results exist for open systems composed of transparent and nondispersive materials, for which the eigenvalue problem is linear. For simple geometries, such as one-dimensional (1D) dielectric cavities or spherically symmetric resonators that mathematically behave as 1D-like systems because each angular momentum is independent of others, it was shown that the QNM set forms a complete and orthogonal basis [29-32]. Recently, the extension of the orthogonality properties to more complex geometries was conjectured (without proof) and checked numerically for photonic crystal cavities [35]. In fact, for three-dimensional (3D) complex geometries that cannot be handled analytically, closing the system with perfectly matched layers [48] allows one to prove the existence of a biorthogonality relation for QNMs of dielectric resonators [43-45]. Furthermore, since the eigenvalue problem is linear, in practice, QNMs can be calculated with linear eigenmode solvers using classical computer libraries [43,44].

In comparison, the use of QNM expansions for the analysis of resonances with absorbing and dispersive materials is in its infancy. For 1D geometries, the completeness of the QNM set has been extended to the absorbing and dispersive case [42]. Recently, using a new approach based on Lorentz reciprocity theorem [49], general theoretical results have been established for arbitrary geometries and materials [45]. In particular, it was shown that QNMs can be normalized, although they no longer form a biorthogonal set as for nondispersive materials. The main consequence is that the singular dominant terms of the QNM expansion can be calculated analytically. These results provided firm theoretical grounds to define a new expression for the mode volume valid for dissipative and dispersive resonators and to revisit the classical formula for the Purcell factor, which has been shown to be an asymptotic expression only valid for large quality factors [45,50]. Because of energy dissipation, the mode volume is a complex quantity, whose imaginary part gives rise to a non-Lorentzian spectral behavior for the LDOS of plasmonic resonators [45]. Using these initial theoretical results, Bai and coworkers derived a general and simple procedure that can be used with any fully vectorial electromagnetic software to calculate and normalize the QNMs of virtually any geometry and material. The procedure has been successfully applied to derive accurate semianalytical expressions of the absorption and scattering cross sections of plasmonic nanoparticles and nanoresonators [46].

The objective of this article is to extend the modal theory proposed in $[45,46]$ for deriving a semianalytical expression of the CDOS for a 3D resonant system with any complex geometry that includes radiative leakage (open system), absorption, and material dispersion. Deriving a generalized form of Eq. (2) is of fundamental importance since such a closed-form expression allows one to quantitatively describe the physics of a complex photonic or plasmonic system with only a few physically meaningful resonances. In particular, it provides an analytical relation between the intrinsic spatial coherence of a complex system and the spatial extent of its eigenmodes.

In Sec. II, we first recall the definition of the eigenmodes of a dissipative resonator (QNMs) and we briefly summarize our recent results on the use of QNMs to represent the optical resonances in 3D dissipative systems $[45,46]$. Then we derive an approximate modal expansion of the CDOS, which is based on the following ansatz: a small discrete set of modes is sufficient to accurately describe the electromagnetic field in the vicinity of the resonator. This assumption is then validated by comparing the analytical predictions to fully vectorial numerical calculations for various examples of increasing complexity. We consider in Sec. III an open but nonabsorbing system composed of two coupled photoniccrystal microcavities. In Sec. IV we test the accuracy of the modal expansion of the CDOS for different plasmonic nanoantennas with radiative leakage, absorption, and material dispersion. Section V summarizes the main conclusions.

\section{MODAL EXPANSION OF THE CDOS IN A DISSIPATIVE SYSTEM}

Wave evolution in closed (or periodic) systems free from absorption and dispersion is routinely analyzed in terms of the eigenmodes associated to the structure. A conservative system is described by a Hermitian (self-adjoint) operator, whose eigenstates are the so-called normal modes [21]. The latter form a complete and orthogonal set in the sense of the energy, meaning that the energy of a superposition of normal modes is equal to the sum of the energies contained in each mode. Thus the system response to external stimuli or perturbations can 
be written with closed-form expressions relying on a modal expansion [21,51]. This is the essence of Eq. (2).

Generalizing Eq. (2) to open, absorbing, and dispersive systems that are obviously not Hermitian is not a trivial task. In order to correctly take into account the presence of radiative leakage, absorption, and material dispersion, one has to explicitly consider the non-Hermitian character of the system and to use its natural modes, which are QNMs with complex eigenfrequencies. In Sec. II A, we first recall the definition of QNMs. In Sec. II B, we then use the properties of QNM expansions derived in [45] and [46] to expand the Green tensor of a dissipative and dispersive system onto a small discrete set of QNMs. Finally, we derive an analytical modal expansion of the CDOS in Sec. II C.

\section{A. Quasinormal modes of a dissipative system}

We consider a resonant system composed of an assembly of absorptive and dispersive nanostructures with open boundaries. The system is characterized by the position- and frequency-dependent permittivity and permeability tensors $\epsilon(\mathbf{r}, \omega)$ and $\mu(\mathbf{r}, \omega)$. Note that the materials can be anisotropic and magnetic; the sole assumption is that the materials are reciprocal, $\epsilon=\epsilon^{T}$ and $\mu=\mu^{T}$, where the superscript ${ }^{T}$ denotes matrix transposition. This system supports QNMs which are the electromagnetic field distributions $\left(\tilde{\mathbf{E}}_{m}, \tilde{\mathbf{H}}_{m}\right)$ that are solutions of Maxwell's equations in the absence of a source,

$$
\begin{aligned}
\nabla \times \tilde{\mathbf{E}}_{m} & =i \tilde{\omega}_{m} \mu\left(\mathbf{r}, \tilde{\omega}_{m}\right) \tilde{\mathbf{H}}_{m}, \\
\nabla \times \tilde{\mathbf{H}}_{m} & =-i \tilde{\omega}_{m} \epsilon\left(\mathbf{r}, \tilde{\omega}_{m}\right) \tilde{\mathbf{E}}_{m},
\end{aligned}
$$

and that satisfy outgoing wave boundary conditions (the Sommerfeld radiation condition as $|\mathbf{r}| \rightarrow \infty)$. Because the energy leaks out or is absorbed, the QNMs possess a finite lifetime $\tau_{m}$ and their eigenfrequency $\tilde{\omega}_{m}$ is complex, with a negative imaginary part $\operatorname{Im}\left(\tilde{\omega}_{m}\right)=-1 / \tau_{m}$ [we use the $\exp (-i \omega t)$ notation for the time-harmonic fields]. The complex frequency defines the quality factor of the resonance $Q_{m}=-\operatorname{Re}\left(\tilde{\omega}_{m}\right) /\left[2 \operatorname{Im}\left(\tilde{\omega}_{m}\right)\right]$. It can also be attached to the usual physical interpretation based on energy balance arguments on the energy stored and dissipated at resonance if the Ohmic losses are small [52]. Note that a tilde is used hereafter to denote complex quantities that are related to the QNMs, such as their fields and frequencies.

Despite the widespread use of optical resonances for various designs, a difficulty arises when handling the QNMs in practice. Actually, because energy is continuously lost, the QNM amplitudes decay in time and $\operatorname{Im}\left(\tilde{\omega}_{m}\right)<0$. As a consequence, the wave vector is also complex, with a negative imaginary part, and since the boundary conditions impose outgoing spherical waves $\exp \left(i \tilde{k}_{m} r\right) / r$ at infinity, the field diverges exponentially as $|\mathbf{r}| \rightarrow \infty[29,30,45]$.

This divergence poses serious problems when attempting to derive a self-consistent formalism capable of modeling how incident waves exchange their energy with the QNMs. In particular, the divergence prevents normalization of the QNM energy, which is infinite. Moreover, the QNMs are not orthogonal in the sense of the energy as the normal modes of Hermitian systems. A different inner product, which is not positive definite, has to be used [45]. The main difference from Hermitian systems is that the QNM norm is a complex number.

\section{B. Modal representation of the field}

In order to generalize Eq. (2) to open, absorbing, and dispersive systems, we need to expand the Green tensor on the discrete set of QNMs supported by the structure. This can be done by using the properties of QNM expansions derived in $[45,46]$. In this section we briefly recall these results before deriving a modal expansion of the CDOS in Sec. II C.

Let us consider the general problem of the radiation by an electric current source $\mathbf{j}(\mathbf{r})$ located in the vicinity of the resonant system. The electromagnetic field $(\mathbf{E}, \mathbf{H})$ radiated by the source at frequency $\omega$ is the solution of Maxwell's equations in the presence of the structure:

$$
\begin{aligned}
\nabla \times \mathbf{E} & =i \omega \mu(\mathbf{r}, \omega) \mathbf{H}, \\
\nabla \times \mathbf{H} & =-i \omega \epsilon(\mathbf{r}, \omega) \mathbf{E}+\mathbf{j}(\mathbf{r}) .
\end{aligned}
$$

We assume that the total field $(\mathbf{E}, \mathbf{H})$ can be expanded as a superposition of QNMs,

$$
\begin{aligned}
\mathbf{E}(\mathbf{r}, \omega) & \approx \sum_{m} \alpha_{m}(\omega) \tilde{\mathbf{E}}_{m}(\mathbf{r}), \\
\mathbf{H}(\mathbf{r}, \omega) & \approx \sum_{m} \alpha_{m}(\omega) \tilde{\mathbf{H}}_{m}(\mathbf{r}),
\end{aligned}
$$

where $\alpha_{m}$ is a complex coefficient that quantifies the coupling of the source to the $m$ th QNM. By applying the general form of the Lorentz reciprocity theorem [49] to Eqs. (3) and (4), it was shown that the QNMs can be normalized and that $\alpha_{m}$ can be written as [45]

$$
\alpha_{m}(\omega)=-i \frac{\int \mathbf{j}(\mathbf{r}) \cdot \tilde{\mathbf{E}}_{m}(\mathbf{r}) d^{3} \mathbf{r}}{2 \epsilon_{0}\left(\omega-\tilde{\omega}_{m}\right)}+f_{m}(\omega),
$$

where $f_{m}(\omega)$ is a nonresonant background that becomes negligible as $\omega \rightarrow \tilde{\omega}_{m}$. The background term comes from the fact that QNMs of dispersive media are not orthogonal. Only in the particular case of nondispersive media are the QNMs orthogonal and $f_{m}(\omega)=0$ [45]. Equation (6) is valid provided that the QNMs obey the normalization condition

$$
\int\left[\tilde{\mathbf{E}}_{m} \cdot \frac{\partial(\omega \epsilon)}{\partial \omega} \tilde{\mathbf{E}}_{m}-\tilde{\mathbf{H}}_{m} \cdot \frac{\partial(\omega \mu)}{\partial \omega} \tilde{\mathbf{H}}_{m}\right] \mathrm{d}^{3} \mathbf{r}=2 \epsilon_{0},
$$

where $\epsilon_{0}$ is the vacuum permittivity and the derivatives are taken at $\omega=\tilde{\omega}_{m}$. Concerning the QNM normalization, the divergence at $|\mathbf{r}| \rightarrow \infty$ is safely handled either by transforming the QNMs, which are formally scattering or continuum states, into square-integrable states with classically available perfectly matched layers [45], or by using a very efficient and general method that relies on the fact that, for $\omega$ close to $\tilde{\omega}_{m}$, any field grows up as $1 /\left(\omega-\tilde{\omega}_{m}\right)$ [46]. The value of the integral in Eq. (7) is chosen to recover the usual normalization condition of normal modes in the particular case of lossless and nondispersive systems.

Before using these results to derive a modal expansion of the CDOS, let us discuss the validity of our key assumption, namely, the modal representation of the field given by Eq. (5). Perhaps the most important issue is the completeness of the QNM set. The completeness of the QNM set has only been 
demonstrated for a limited number of simple situations, for 1D [29,30,42] or spherical [31,32] open cavities, for which it has been established that a QNM expansion is complete inside the cavity. However, in the general 3D case, the completeness remains an open question. In particular, a QNM expansion cannot be used to represent the far field outside the resonator, especially if the excitation source is located there (as in the case of plane-wave illumination). This is the reason why in [46], the scattered field (i.e., the system response), and not the total field, has been represented as a QNM superposition, $\mathbf{E}=\mathbf{E}_{i}+\sum_{m} \beta_{m} \tilde{\mathbf{E}}_{m}$, where $\mathbf{E}_{i}$ is the field radiated by the source $\mathbf{j}(\mathbf{r})$ in the absence of the resonator. Such a field representation allowed us to derive accurate semianalytical expressions of the absorption and scattering cross sections of plasmonic nanoparticles [46].

However, in order to maintain full analyticity in the CDOS derivation and to obtain a simple expression that reduces to Eq. (2) when energy dissipation vanishes, we assume hereafter (as in [45]) that the total field can actually be represented as a QNM superposition. Through numerical calculations, we illustrate in Secs. III and IV that this assumption provides very accurate predictions for the CDOS in many practical cases of interest.

\section{Modal expansion of the CDOS}

For deriving the CDOS, we are interested in the electric Green tensor $\mathbf{G}\left(\mathbf{r}, \mathbf{r}^{\prime}, \omega\right)$. The latter connects the electric field created at point $\mathbf{r}$ to an electric-dipole source $\mathbf{p}$ located at point $\mathbf{r}^{\prime}$ through the relation $\mathbf{E}(\mathbf{r}, \omega)=\mu_{0} \omega^{2} \mathbf{G}\left(\mathbf{r}, \mathbf{r}^{\prime}, \omega\right) \mathbf{p}$. Therefore, the source $\mathbf{j}(\mathbf{r})$ appearing in Eq. (6) has to be replaced by the current distribution associated with a point dipole, $\mathbf{j}(\mathbf{r})=-i \omega \mathbf{p} \delta\left(\mathbf{r}-\mathbf{r}^{\prime}\right)$. We further assume that the nonresonant contribution $f_{m}(\omega)$ to the coupling coefficient $\alpha_{m}$ in dispersive systems can be neglected. If the system response is essentially driven by a single mode, this assumption is clearly valid provided that the frequency $\omega$ is close to the corresponding eigenfrequency $\operatorname{Re}\left(\tilde{\omega}_{m}\right)$. If now several modes significantly contribute to the system response, the assumption amounts to considering that these QNMs are mutually orthogonal. This is valid provided that the variation of $\epsilon$ and $\mu$ over the spectral range that includes the few eigenfrequencies of interest can be accurately described by a first-order Taylor expansion.

In this case and for a point source, Eq. (6) becomes

$$
\alpha_{m}\left(\mathbf{r}^{\prime}, \omega\right)=-\frac{\omega \mathbf{p} \cdot \tilde{\mathbf{E}}_{m}\left(\mathbf{r}^{\prime}\right)}{2 \epsilon_{0}\left(\omega-\tilde{\omega}_{m}\right)} .
$$

By using the modal expansion of the electric field given by Eq. (5) and the expression of the coupling coefficients given by Eq. (8), we obtain an approximate closed-form expression for the Green tensor of a dissipative system,

$$
\mathbf{G}\left(\mathbf{r}, \mathbf{r}^{\prime}, \omega\right) \approx-\frac{c^{2}}{2 \omega} \sum_{m} \frac{\tilde{\mathbf{E}}_{m}(\mathbf{r}) \otimes \tilde{\mathbf{E}}_{m}\left(\mathbf{r}^{\prime}\right)}{\omega-\tilde{\omega}_{m}} .
$$

Note that the divergence of the real part at $\mathbf{r}=\mathbf{r}^{\prime}$ is not correctly accounted for by Eq. (9), which is in practice the finite sum of a few regular terms at $\mathbf{r}=\mathbf{r}^{\prime}$. This limitation may be circumvented by using the scattering formulation in [46], as discussed in Sec. II B. However, since we are interested in the CDOS defined by Eq. (1), hereafter we only need the imaginary part of the Green tensor and we can safely use Eq. (9).

By using the modal expansion of the Green tensor given by Eq. (9), the CDOS defined in Eq. (1) is straightforwardly expanded onto the set of QNMs supported by the dissipative resonant system:

$$
\rho\left(\mathbf{r}, \mathbf{r}^{\prime}, \omega\right)=-\frac{1}{\pi} \sum_{m} \operatorname{Im}\left[\frac{\operatorname{Tr} \tilde{\mathbf{E}}_{m}(\mathbf{r}) \otimes \tilde{\mathbf{E}}_{m}\left(\mathbf{r}^{\prime}\right)}{\omega-\tilde{\omega}_{m}}\right] .
$$

The CDOS is the sum of three terms that correspond to the three directions of space, $\rho=\rho_{x x}+\rho_{y y}+\rho_{z z}$, and simple derivations lead to

$$
\begin{aligned}
\rho_{i i}\left(\mathbf{r}, \mathbf{r}^{\prime}, \omega\right)= & \frac{1}{\pi} \sum_{m} \frac{\omega_{m}}{2 Q_{m}} \frac{\operatorname{Re}\left[\tilde{E}_{m i}(\mathbf{r}) \tilde{E}_{m i}\left(\mathbf{r}^{\prime}\right)\right]}{\left(\omega-\omega_{m}\right)^{2}+\left(\frac{\omega_{m}}{2 Q_{m}}\right)^{2}} \\
& \times\left[1-2 Q_{m} \frac{\omega-\omega_{m}}{\omega_{m}} \frac{\operatorname{Im}\left[\tilde{E}_{m i}(\mathbf{r}) \tilde{E}_{m i}\left(\mathbf{r}^{\prime}\right)\right]}{\operatorname{Re}\left[\tilde{E}_{m i}(\mathbf{r}) \tilde{E}_{m i}\left(\mathbf{r}^{\prime}\right)\right]}\right],
\end{aligned}
$$

with $\quad i=x, y, z, \quad \omega_{m}=\operatorname{Re}\left(\tilde{\omega}_{m}\right), \quad$ and $\quad Q_{m}=$ $-\operatorname{Re}\left(\tilde{\omega}_{m}\right) /\left[2 \operatorname{Im}\left(\tilde{\omega}_{m}\right)\right]$. Equation (11) provides a fully analytical expression of the CDOS that only involves the QNMs. It represents the generalization of Eq. (2) in the presence of energy dissipation. Indeed, for a conservative system $\left(Q_{m} \rightarrow \infty\right)$, the QNM-field components are either real or purely imaginary and we can use the mathematical identity $\lim _{\gamma_{m} \rightarrow 0} \gamma_{m} /\left[\left(\omega-\omega_{m}\right)^{2}+\gamma_{m}^{2}\right]=\pi \delta\left(\omega-\omega_{m}\right)$ to show that Eq. (11) reduces to Eq. (2), which then appears to be valid in the limit of large quality factors. We emphasize that the presence of dissipation does not come out only as a widening of the Dirac $\delta$ function into a Lorentzian line shape with a quality factor $Q_{m}$. Indeed, because of the second term in the brackets, which is proportional to $\omega-\omega_{m}$, the spectral variation of the CDOS can be more complex than a simple Lorentzian line shape, a surprising property already observed in [45] for the LDOS. Finally, we note that Eq. (11) for $\mathbf{r}=\mathbf{r}^{\prime}$ is consistent with the expression of the LDOS derived in [45].

Equation (11) is of fundamental importance since it quantifies the link between the CDOS, which gives a measure of the intrinsic spatial coherence [20], and the optical modes supported by the system. The CDOS sums up the contributions of all eigenmodes that connect $\mathbf{r}$ to $\mathbf{r}^{\prime}$, with possibly a complex spectral variation. We emphasize that the individual contribution of a given mode can be negative, depending on its field distribution. A high CDOS (in absolute value) means that the system supports at least one mode that connects $\mathbf{r}$ to $\mathbf{r}^{\prime}$. However, a weak CDOS can have two different physical origins. Either the system does not support any mode that connects both points or, on the contrary, both points are connected by several modes that interfere destructively so that their contributions to the total CDOS cancel each other. As a consequence, only the calculation of the QNMs can provide a sound interpretation of the lack of coherence between two points in the system. In the following sections, we illustrate these conclusions and we check the accuracy of Eq. (11) in several examples that are representative of modern studies in nanophotonics. 
Before applying the theoretical results to concrete examples, we present the numerical implementation of the theory. The technique that we use to calculate a QNM relies on an iterative solving of Maxwell's equations in the complex frequency plane [46]. The system is excited with a source (a plane wave, a dipole source located nearby, etc.) at a complex frequency $\omega$ close to the QNM frequency $\tilde{\omega}_{m}$, some quantity representative of the system response is calculated, and the excitation frequency $\omega$ is iteratively changed in the complex plane until the response diverges. After a few iterations, the frequency $\omega$ tends towards $\tilde{\omega}_{m}$ and the scattered field tends toward the QNM field. Material dispersion can be easily taken into account with an analytical continuation of the permittivity in the complex frequency plane. In this work, we have used a dipole source to excite the resonance. The iterative approach used for the pole calculation has been detailed in Appendix 2 of Ref. [46]. After the pole is found, the QNM fields $\tilde{\mathbf{E}}_{m}$ and $\tilde{\mathbf{H}}_{m}$ are calculated for a complex frequency close to $\tilde{\omega}_{m}$ in order to avoid the singularity at $\omega=\tilde{\omega}_{m}$ and the QNMs are normalized with the technique proposed in [46].

The iterative calculation of a QNM can be realized with virtually any frequency-domain numerical method. For instance, commercial software such as COMSOL Multiphysics can be used [46]. Hereafter, we use a numerical method known as the aperiodic Fourier modal method (a-FMM), implemented either in Cartesian [53] or in cylindrical coordinates [54]. The method is an extension to aperiodic structures of the usual FMM, which is also called rigorous coupled-wave analysis (RCWA) $[55,56]$. The a-FMM relies on a semianalytical integration of Maxwell's equations in one space direction and on the use of perfectly matched layers and a discretization of the electromagnetic field in the Fourier space in the other directions. In Secs. III and IV, we compare the theoretical predictions given by the modal expansion in Eq. (11) with a rigorous calculation of the full Green tensor. The latter is also performed with the a-FMM [57].

\section{COUPLED PHOTONIC CRYSTAL MICROCAVITIES}

In order to test the modal expansion, we first consider a photonic-crystal coupled-cavity system. Neither absorption nor material dispersion is present in the system, but it allows us to illustrate the peculiar spectral variation of the CDOS and its cancellation when two modes interfere destructively. The system, whose LDOS was initially investigated in [22] and [23], consists of two cavities that are formed by removing two rods in a finite-size $2 \mathrm{D}$ photonic crystal made of a square array (period $d$ ) of $9 \times 10$ semiconductor rods (radius $r=0.2 d$ and refractive index $n=3$ ) in air (see Fig. 1). We consider the transverse electric polarization with an electric field parallel to the cylinders, $\mathbf{E}=E \mathbf{z}$. For this case of polarization, the CDOS reduces to a single term, $\rho_{z z}$.

The dominant QNMs have been calculated with the a-FMM [53]. Their intensity distributions $\left|\tilde{E}_{z}\right|^{2}$ are superimposed on the geometry in Fig. 1. Because of the proximity to the photonic-crystal boundary, the mode that is mainly localized in the upper cavity (labeled 2) has a low $Q$ factor limited by leakage into the air clad $\left(Q_{2}=90\right)$, whereas the other mode (localized in the bottom cavity and labeled 1) has a higher $Q$ factor $\left(Q_{1}=310\right)$. The normalized complex eigenfrequencies
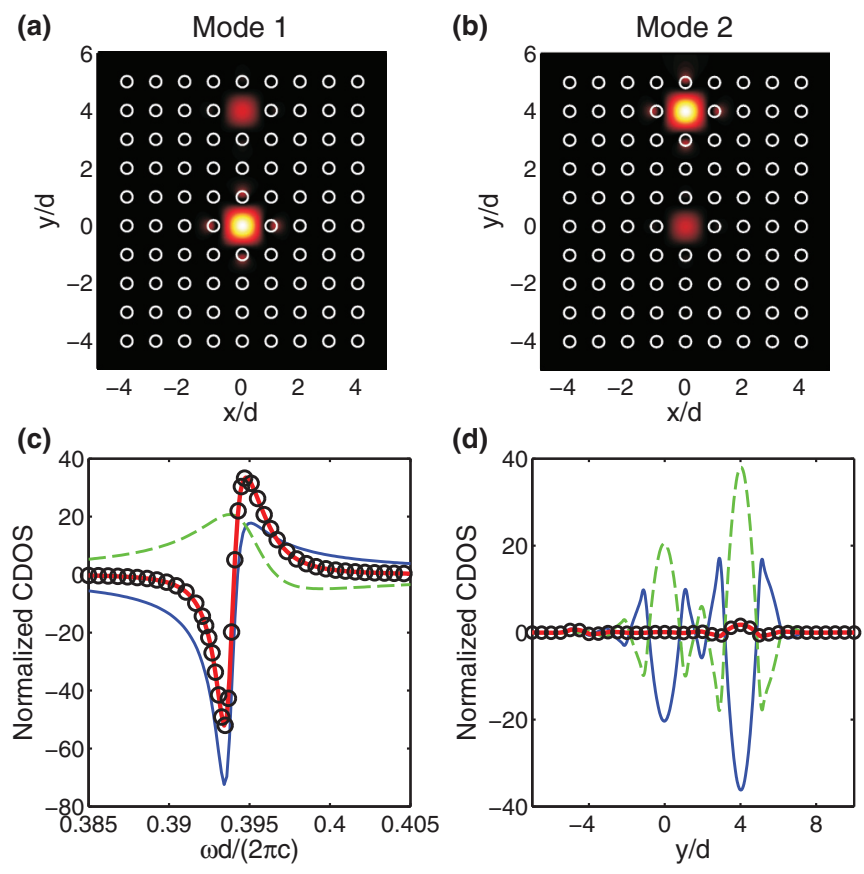

FIG. 1. (Color online) Coupled photonic-crystal microcavities. Two cavities are formed by removing two cylinders in a finite-size photonic crystal. (a, b) Intensity distributions $\left|\tilde{E}_{z}\right|^{2}$ of the two dominant QNMs. White circles represent the semiconductor cylinders forming the photonic crystal. We calculate the CDOS for $\mathbf{r}^{\prime}$ fixed in the center of the upper cavity; it is normalized by the LDOS in vacuum. (c) Spectral variation of the CDOS for $\mathbf{r}$ fixed in the center of the bottom cavity. The total CDOS results from two modal contributions, $\rho_{1}$ and $\rho_{2}$, given by Eq. (11) and shown by the solid (blue) and dashed (green) curves. The sum [bold solid (red) curve] is in excellent agreement with fully vectorial calculations of the full Green tensor shown by open black circles. (d) Cross section of the CDOS distribution for $\omega d /(2 \pi c)=0.3940$ [frequency where the CDOS in (c) cancels]. Point $\mathbf{r}$ is varied along the $x=0$ axis. For this particular frequency, both modal contributions interfere destructively everywhere.

are $\tilde{\omega}_{1} d /(2 \pi c)=0.3938-0.0006 i$ and $\tilde{\omega}_{2} d /(2 \pi c)=0.3949$ $0.0023 i$.

According to the QNM formalism developed in Sec. II, the CDOS is the sum of two independent contributions, $\rho_{1}$ and $\rho_{2}$, that solely depend on the two QNMs supported by the system [see Eq. (11)]. We first calculated the spectral variation of the CDOS that connects the cavity centers, $\mathbf{r}=(0,0) d$ and $\mathbf{r}^{\prime}=(0,4) d$. Both contributions, $\rho_{1}$ and $\rho_{2}$, are shown, by the solid (blue) and dashed (green) curves in Fig. 1(c), and the bold solid (red) curve is the sum. The predictions of the modal expansion are in quantitative agreement with exact calculations of the full Green function performed with the a-FMM and shown by the open black circles. The difference is smaller than 0.5 over the entire spectrum. In particular, the modal expansion accurately predicts the peculiar spectral variation of the CDOS, which takes both negative and positive values. In the context of the coherence between two emitters, positive and negative values of the CDOS are related to super- and subradiance, respectively.

Far from the system resonances, the CDOS is small because both modal contributions, $\rho_{1}$ and $\rho_{2}$, are vanishing; there is no 
coherence between the centers of the two cavities because there is no mode connecting them. On the contrary, near the system resonances, it is an interference mechanism between both modes that is responsible for the cancellation of the CDOS at $\omega d /(2 \pi c)=0.3940$. Indeed, for this particular frequency, the two modal contributions $\rho_{1}$ and $\rho_{2}$ cancel each other, resulting in an incoherent relation between the centers of the cavities. We also calculated the spatial distribution of the CDOS for this particular frequency and for $\mathbf{r}^{\prime}$ fixed in the center of the upper cavity, $\mathbf{r}^{\prime}=(0,4) d$, the second point $\mathbf{r}$ being varied along the $y$ axis $(x=0)$. The results presented in Fig. 1(d) show that the CDOS remains extremely weak whatever the position along the axis, and this behavior is quantitatively predicted by the modal expansion, with two contributions that are opposite and that almost cancel each other everywhere.

\section{PLASMONIC ANTENNAS COMPOSED OF METALLIC NANORODS}

We now demonstrate the accuracy of the QNM formalism with a more complex system including absorption and dispersion in addition to radiative leakage. We consider resonant plasmonic antennas made of metallic nanorods. We begin with a single nanorod (see Fig. 2), a system that has recently received considerable attention for control of the spontaneous emission [58-60]. We further validate the predictions of Eq. (11) for a more complex geometry that supports multiple resonances (see Fig. 3).

\section{A. Single metallic nanorod: The dipole nanoantenna}

We consider a gold nanorod with a diameter $D=30 \mathrm{~nm}$ and a length $L=100 \mathrm{~nm}$, which constitutes a classical extension of the half-wave dipolar radio antenna to optical frequencies [61]. The nanorod is embedded in a host medium of refractive index $n=1.5$. In the spectral range of interest, a single QNM is dominant, namely, the dipole-like mode of the nanorod. This mode has been calculated with the a-FMM implemented in cylindrical coordinates [54] and its electric-field distribution is shown in Fig. 2(a). To calculate QNMs in dispersive media, one needs an analytical continuation of the permittivity for complex frequencies. For gold, we have used a Drude model that fits the tabulated data in [62], $\epsilon=1-\omega_{p}^{2} /\left(\omega^{2}+i \omega \gamma\right)$ with $\omega_{p}=1.26 \times 10^{16} \mathrm{~s}^{-1}$ and $\gamma=1.41 \times 10^{14} \mathrm{~s}^{-1}$.

We have used this single mode in Eq. (11) to calculate the $z$ component of the CDOS $\rho_{z z}$ at the resonance frequency $(\lambda=920 \mathrm{~nm})$ for a point $\mathbf{r}^{\prime}$ fixed on the axis at a $10-\mathrm{nm}$ distance above the nanorod, the second point $\mathbf{r}$ being varied, either along the $z$ axis with $x=0$ [Fig. 2(b)] or along the $x$-axis with $z=0$ [Fig. 2(c)]. In both cases, the predictions of the modal expansion [solid (red) curves] are in quantitative agreement with the exact calculations of the full Green tensor (open black circles obtained with the a-FMM). The same agreement is obtained for different frequencies and different positions in the vicinity of the nanorod. For distances larger than $150 \mathrm{~nm}$, the resonance is only weakly excited and the validity of representing the Green tensor solely with the nanorod modes becomes questionable; see the discussion in Sec. II B and in $[45,46]$.
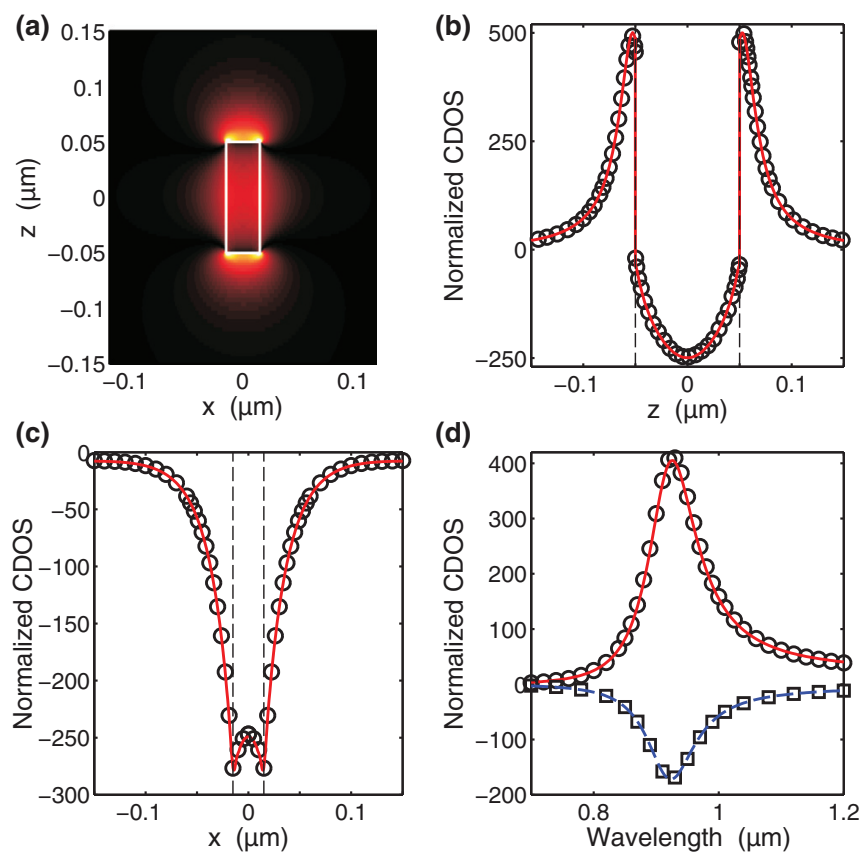

(d)

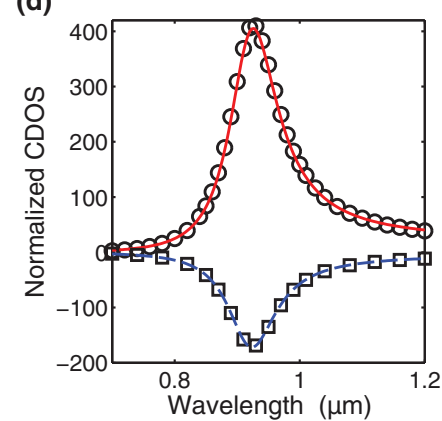

FIG. 2. (Color online) Single gold nanorod (diameter $D=$ $30 \mathrm{~nm}$, length $L=100 \mathrm{~nm}$ ) embedded in a host medium of refractive index $n=1.5$. (a) Electric-field distribution $\left|\tilde{E}_{z}\right|$ of the dipole-like QNM (complex frequency $2 \pi c / \tilde{\omega}=920+47 i \mathrm{~nm}$ ). We calculate the CDOS for $\mathbf{r}^{\prime}$ fixed on the axis at a 10-nm distance above the nanorod; it is normalized by the LDOS in a bulk material with $n=1.5$. (b) Longitudinal cross section of the $z$ component of the CDOS at nanorod resonance $\lambda=920 \mathrm{~nm}$. Point $\mathbf{r}$ is moved along the rod axis. (c) Transversal cross section of the $z$ component of the CDOS at $\lambda=920 \mathrm{~nm}$. Point $\mathbf{r}$ is now moved perpendicularly to the rod axis at $z=0$. Dashed vertical lines represent the nanorod boundaries. (d) Spectral variation of the CDOS. In (b)-(d), solid and dashed curves are obtained from Eq. (11) for the QNM shown in (a) and the markers are fully vectorial calculations of the full Green tensor.

We have also calculated the spectral variation of the CDOS for different fixed positions [see Fig. 2(d)]. The point $\mathbf{r}^{\prime}$ is again fixed on the axis at a 10 -nm distance above the nanorod. The second point $\mathbf{r}$ is fixed either on the axis at a $10-\mathrm{nm}$ distance below the nanorod [solid (red) curve and open black circles] or off the axis at $x=D / 2+10 \mathrm{~nm}$ and $z=0$ [dashed (blue) curve and open black squares]. The predictions of the modal expansion, given by the solid and dashed curves, are in good agreement with exact calculations given by the markers. For both positions of the point $\mathbf{r}$, the coherence is maximum at resonance. Two points located on the nanorod axis are linked by a positive CDOS; they are coherent and give rise to constructive interferences. On the contrary, mixing on-axis and off-axis points gives rise to destructive interferences since these points are linked by a negative CDOS.

\section{B. Complex nanoantenna made of three metallic nanorods}

As the final example we consider an optical antenna made of three aligned gold nanorods that are identical to the one studied in the previous section. The nanorods are separated by small gaps, $g=40 \mathrm{~nm}$ [see Fig. 3(a)]. The 
(a)

M1

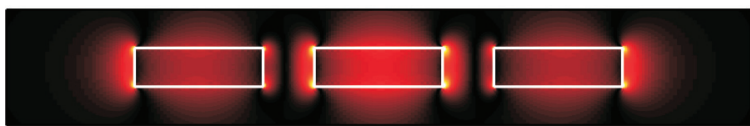

M2

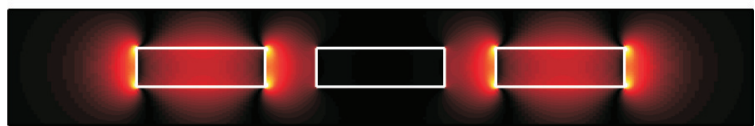

M3

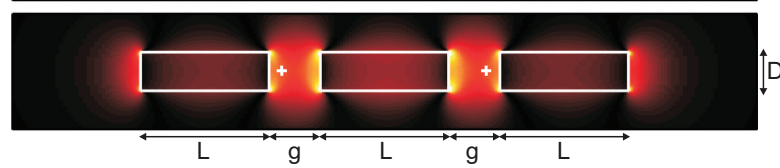

(b)

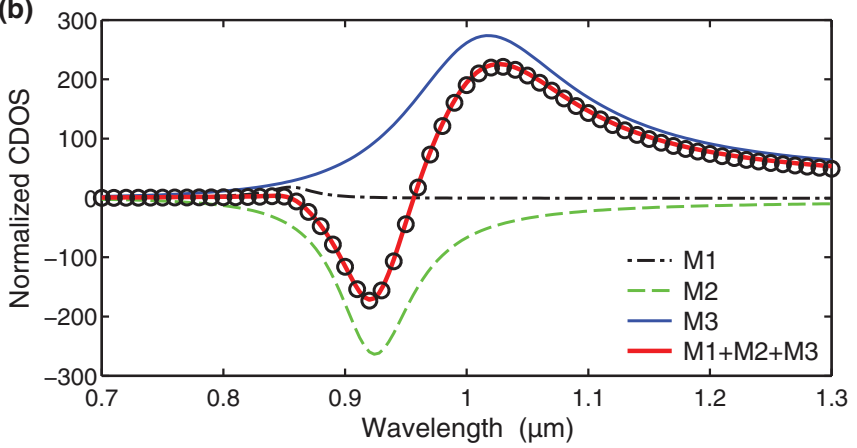

FIG. 3. (Color online) Three identical gold nanorods (diameter $D=30 \mathrm{~nm}$, length $L=100 \mathrm{~nm}$ ) embedded in a host medium of refractive index $n=1.5$. The rods are aligned and separated by a small gap, $g=40 \mathrm{~nm}$. (a) Electric-field distributions $\left|\tilde{E}_{z}\right|$ of the three QNMs that result from the coupling of the three individual dipole-like modes. (b) Spectral variation of the CDOS. Points $\mathbf{r}$ and $\mathbf{r}^{\prime}$ are fixed at the positions marked by the white crosses in (a) (on the axis, inside the gaps, at a 10 -nm distance from the left and right nanorods) and the CDOS is normalized by the LDOS in a bulk material with $n=1.5$. The three modal contributions to the CDOS are shown by the dashed-dotted black, dashed (green), and solid (blue) curves. The bold solid (red) curve is the sum and the open black circles show the results of fully vectorial calculations.

proximity of the nanorods gives rise to a coupling between the dipole-like modes supported by each individual cylinder, and the complete structure supports three dominant QNMs, whose electric-field distributions are shown in Fig. 3(a). The corresponding complex eigenfrequencies (from top to bottom) are $2 \pi c / \tilde{\omega}_{1}=857+23 i \mathrm{~nm}, 2 \pi c / \tilde{\omega}_{2}=922+37 i \mathrm{~nm}$, and $2 \pi c / \tilde{\omega}_{3}=1001+82 i \mathrm{~nm}$.

The three contributions to the $z$ component of the CDOS $\rho_{z z}$ are calculated with Eq. (11) as a function of the frequency for fixed positions $\mathbf{r}$ and $\mathbf{r}^{\prime}$. Points $\mathbf{r}$ and $\mathbf{r}^{\prime}$ [shown by the white crosses in Fig. 3(a)] are located inside the gaps, on the system axis, at a 10-nm distance from the left and the right nanorods, respectively. The three contributions to the total CDOS are represented in Fig. 3(b) by the dasheddotted (black), the dashed (green), and the solid (blue) curves. The bold solid (red) curve is the sum. The predictions of the modal expansion are in quantitative agreement with the exact calculations of the full Green function shown by the open black circles. In particular, we note that the assumption made on the QNM orthogonality [see Eq. (8)] does not reduce the accuracy.

We emphasize that Eq. (11) accurately predicts the peculiar spectral variation of the CDOS, which alternatively takes positive and negative values. The modal expansion allows us to get a precise physical insight into the spatial coherence of the system. Mode 1 (dashed-dotted black curve) contributes only weakly to the CDOS because the points $\mathbf{r}$ and $\mathbf{r}^{\prime}$ that have been chosen are weakly connected through this mode. On the contrary, mode 2 and mode 3 strongly connect these points. The contribution of mode 2 gives rise to destructive interferences [negative CDOS; dashed (green) curve], whereas the contribution of mode 3 gives rise to constructive interferences [positive CDOS; solid (blue) curve]. As a result, the total CDOS depends strongly on the frequency and can be either positive or negative.

\section{CONCLUSION}

We have derived a modal expansion of the imaginary part of the Green tensor that is valid for 3D dissipative resonant systems with any complex geometry. The theory, which relies on the concept of QNMs with complex frequencies, provides an accurate closed-form expression of the CDOS for systems with radiative leakage, absorption, and dispersion. Such a modal expansion is extremely useful, as it analytically highlights the link between the optical modes supported by a resonant system and the spatial coherence of the electromagnetic field. Once a few dominant modes have been calculated, any spectral or spatial variation is treated analytically, in contrast to full numerical methods. We believe that the present theoretical work may impact many fields of photonics and plasmonics, where spatial coherence plays an important role. We also expect that similar modal representations may be useful for other waves, such as acoustic or quantum waves.

\section{ACKNOWLEDGMENTS}

The authors acknowledge A. Cazé and R. Pierrat for fruitful discussions.
[1] J. N. Anker, W. P. Hall, O. Lyandres, N. C. Shah, J. Zhao, and R. P. van Duyne, Nat. Mater. 7, 442 (2008).

[2] N. Liu, M. L. Tang, M. Hentschel, H. Giessen, and A. P. Alivisatos, Nat. Mater. 10, 631 (2011).

[3] X. Li and M. I. Stockman, Phys. Rev. B 77, 195109 (2008).
[4] A. Sentenac and P. C. Chaumet, Phys. Rev. Lett. 101, 013901 (2008)

[5] R. F. Oulton, V. J. Sorger, T. Zentgraf, R. M. Ma, C. Gladden, L. Dai, G. Bartal, and X. Zhang, Nature (London) 461, 629 (2009); M. A. Noginov, G. Zhu, A. M. Belgrave, R. Bakker, V. M. Shalaev, E. E. Narimanev, S. Stout, E. Herz, T. Suteewong, and U. Wiesner, ibid. 460, 1110 (2009). 
[6] M. Khajavikhan, A. Simic, M. Katz, J. H. Lee, B. Slutsky, A. Mizrahi, V. Lomakin, and Y. Fainman, Nature (London) 482, 204 (2012).

[7] A. J. Shields, Nature Photon. 1, 215 (2007).

[8] J. Claudon, J. Bleuse, N. S. Malik, M. Bazin, P. Jaffrennou, N. Gregersen, C. Sauvan, P. Lalanne, and J.-M. Gérard, Nature Photon. 4, 174 (2010).

[9] H. A. Atwater and A. Polman, Nat. Mater. 9, 205 (2010).

[10] M. Durach, A. Rusina, and M. I. Stockman, Nano Lett. 7, 3145 (2007).

[11] R. H. Dicke, Phys. Rev. 93, 99 (1954).

[12] V. N. Pustovit and T. V. Shahbazyan, Phys. Rev. Lett. 102, 077401 (2009).

[13] D. Martín-Cano, L. Martín-Moreno, F. J. García-Vidal, and E. Moreno, Nano Lett. 10, 3129 (2010).

[14] J. de Rosny and M. Fink, Phys. Rev. Lett. 89, 124301 (2002).

[15] S. A. Ponomarenko and E. Wolf, Phys. Rev. E 65, 016602 (2001).

[16] R. L. Weaver and O. I. Lobkis, Phys. Rev. Lett. 87, 134301 (2001).

[17] T. Setälä, K. Blomstedt, M. Kaivola, and A. T. Friberg, Phys. Rev. E 67, 026613 (2003).

[18] K. Joulain, J.-P. Mulet, F. Marquier, R. Carminati, and J.-J. Greffet, Surf. Sci. Rep. 57, 59 (2005).

[19] R. Carminati, R. Pierrat, J. de Rosny, and M. Fink, Opt. Lett. 32, 3107 (2007).

[20] A. Cazé, R. Pierrat, and R. Carminati, Phys. Rev. Lett. 110, 063903 (2013).

[21] P. M. Morse and H. Feshbach, Methods of Theoretical Physics (McGraw-Hill, New York, 1953).

[22] M. M. Dignam, D. P. Fussell, M. J. Steel, C. M. de Sterke, and R. C. McPhedran, Phys. Rev. Lett. 96, 103902 (2006).

[23] D. P. Fussell, M. M. Dignam, M. J. Steel, C. M. de Sterke, and R. C. McPhedran, Phys. Rev. A 74, 043806 (2006).

[24] A. F. Koenderink, Opt. Lett. 35, 4208 (2010).

[25] A. J. F. Siegert, Phys. Rev. 56, 750 (1939).

[26] R. M. More, Phys. Rev. A 4, 1782 (1971).

[27] R. M. More and E. Gerjuoy, Phys. Rev. A 7, 1288 (1973).

[28] B. J. Hoenders, J. Math. Phys. 20, 329 (1979).

[29] P. T. Leung, S. Y. Liu, and K. Young, Phys. Rev. A 49, 3057 (1994). P. T. Leung, S. Y. Liu, S. S. Tong, and K. Young, ibid. 49, 3068 (1994).

[30] R. K. Chang and A. J. Campillo, Optical Processes in Microcavities (World Scientific, London, 1996), Chap. 1.

[31] K. M. Lee, P. T. Leung, and K. M. Pang, J. Opt. Soc. Am. B 16, 1409 (1999).

[32] P. T. Leung, K. M. Pang, and K. Young, J. Phys. A: Math. Gen. 39, 247 (2006).

[33] A. Settimi, S. Severini, N. Mattiucci, C. Sibilia, M. Centini, G. D’Aguanno, M. Bertolotti, M. Scalora, M. Bloemer, and C. M. Bowden, Phys. Rev. E 68, 026614 (2003).

[34] M. B. Doost, W. Langbein, and E. A. Muljarov, Phys. Rev. A 85, 023835 (2012).

[35] P. T. Kristensen, C. van Vlack, and S. Hughes, Opt. Lett. 37, 1649 (2012).
[36] S. Derom, R. Vincent, A. Bouhelier, and G. Colas des Francs, Europhys. Lett. 98, 47008 (2012).

[37] S. Hein, T. Hohage, and W. Koch, J. Fluid. Mech. 506, 255 (2004).

[38] A. W. Snyder and J. D. Love, Optical Waveguide Theory (Chapman \& Hall, London, 1983).

[39] M. I. Stockman, S. V. Faleev, and D. J. Bergman, Phys. Rev. Lett. 87, 167401 (2001).

[40] G. Boudarham and M. Kociak, Phys. Rev. B 85, 245447 (2012).

[41] H. E. Türeci, A. D. Stone, and B. Collier, Phys. Rev. A 74, 043822 (2006).

[42] P. T. Leung, S. Y. Liu, and K. Young, Phys. Rev. A 49, 3982 (1994).

[43] Q. I. Dai, W. C. Chew, Y. H. Lo, Y. G. Liu, and L. J. Jiang, IEEE Antenn. Wireless Propag. Lett. 11, 1052 (2012).

[44] B. Vial, F. Zolla, A. Nicolet, and M. Commandré, Phys. Rev. A 89, 023829 (2014).

[45] C. Sauvan, J.-P. Hugonin, I. S. Maksymov, and P. Lalanne, Phys. Rev. Lett. 110, 237401 (2013).

[46] Q. Bai, M. Perrin, C. Sauvan, J.-P. Hugonin, and P. Lalanne, Opt. Express 21, 27371 (2013).

[47] N. V. Budko and A. B. Samokhin, Phys. Rev. Lett. 96, 023904 (2006).

[48] Stretched-coordinate perfectly matched layers map real-space coordinates to complex numbers, resulting in exponentially decaying waves in the perfectly matched layer region while preserving ougoing wave boundary conditions; see, for instance, W. C. Chew and W. H. Weedon, Microwave Opt. Technol. Letters 7, 599 (1994).

[49] The Lorentz reciprocity theorem is a general theorem that can be directly deduced from Maxwell's equations. It is valid for any system, with radiative leakage, absorption, or dispersion. The only restriction is that the materials must be reciprocal (i.e., the permittivity and permeability tensors must be symmetrical).

[50] M. Agio and D. Martin-Cano, Nature Photon. 7, 674 (2013).

[51] J. D. Joannopoulos, S. G. Johnson, J. N. Winn, and R. D. Meade, Photonic Crystals (Princeton University Press, Princeton, NJ, 2008), Chap. 2.

[52] P. Lalanne, C. Sauvan, and J.-P. Hugonin, Laser Photon. Rev. 2, 514 (2008).

[53] E. Silberstein, P. Lalanne, J.-P. Hugonin, and Q. Cao, J. Opt. Soc. Am. A 18, 2865 (2001).

[54] N. Bonod, E. Popov, and M. Nevière, J. Opt. Soc. Am. A 22, 481 (2005).

[55] M. G. Moharam, E. B. Grann, D. A. Pommet, and T. K. Gaylord, J. Opt. Soc. Am. A 7, 599 (1995).

[56] L. Li, J. Opt. Soc. Am. A 14, 2758 (1997).

[57] G. Lecamp, J. P. Hugonin, and P. Lalanne, Opt. Express 15, 11042 (2007).

[58] P. Mühlschlegel, H.-J. Eisler, O. J. F. Martin, B. Hecht, and D. W. Pohl, Science 308, 1607 (2005).

[59] L. Novotny and N. F. van Hulst, Nature Photon. 5, 83 (2011).

[60] M. Agio, Nanoscale 4, 692 (2012).

[61] L. Novotny, Phys. Rev. Lett. 98, 266802 (2007).

[62] E. D. Palik, Handbook of Optical Constants of Solids (Academic Press, New York, 1985). 